\title{
A COMPLETE STABILITY THEORY FOR THE KIRCHHOFF THIN PLATE UNDER ALL KINDS OF ACTIONS
}

Shyh-Rong Kuo

Department of Harbor and River Engineering, National Taiwan Ocean University, No. 2, Pei-Ning Road, Keelung 20224, Taiwan, R.O.C., srkuo@mail.ntou.edu.tw

Chih-Chang Chi

Computation and Simulation Center, National Taiwan Ocean University, No. 2, Pei-Ning Road, Keelung 20224, Taiwan, R.O.C.

Yeong-Bin Yang Department of Civil Engineering, National Taiwan University, No. 1, Sec. 4, Roosevelt Road, Taipei 10617, Taiwan, R.O.C.

Follow this and additional works at: https://jmstt.ntou.edu.tw/journal

Part of the Civil and Environmental Engineering Commons

\section{Recommended Citation}

Kuo, Shyh-Rong; Chi, Chih-Chang; and Yang, Yeong-Bin (2009) "A COMPLETE STABILITY THEORY FOR THE KIRCHHOFF THIN PLATE UNDER ALL KINDS OF ACTIONS," Journal of Marine Science and Technology. Vol. 17: Iss. 3, Article 4.

DOI: $10.51400 / 2709-6998.1955$

Available at: https://jmstt.ntou.edu.tw/journal/vol17/iss3/4

This Research Article is brought to you for free and open access by Journal of Marine Science and Technology. It has been accepted for inclusion in Journal of Marine Science and Technology by an authorized editor of Journal of Marine Science and Technology. 


\section{A COMPLETE STABILITY THEORY FOR THE KIRCHHOFF THIN PLATE UNDER ALL KINDS OF ACTIONS}

\section{Acknowledgements}

The first author would like to express his thank to the National Science Council, Taiwan for their financial support under Grant Number: NSC 93-2211-E-019-009. In addition, his thank also goes to Prof. Weichung Yeih and Prof. JinagRen Chang for some valuable discussions on this work. 


\title{
A COMPLETE STABILITY THEORY FOR THE KIRCHHOFF THIN PLATE UNDER ALL KINDS OF ACTIONS
}

\author{
Shyh-Rong Kuo*, Chih-Chang Chi**, and Yeong-Bin Yang***
}

Key words: rigid body rule, structural stability, virtual work equation, plates, Kirchhoff's force.

\begin{abstract}
A complete stability theory for a plate can be constructed by an incremental virtual work equation describing instability effects induced by all kinds of actions. Besides, this incremental virtual work equation should satisfy the rigid body rule, i.e., it should objectively obey the rigid body rule no matter what coordinates systems are adopted. In this paper, a complete nonlinear stability theory for the Kirchhoff thin plate is proposed by using the principle of virtual work and the update Lagrangian formulation. Then, a rigid body motion testing method is developed for examining the incremental virtual work equation. In developing such a theory, three key procedures are especially considered. First of all, the virtual strain energy contributed from all six nonlinear strain components are clearly identified and then, two actions on the effective transverse edge per unit length, namely the Kirchhoff's forces and moment per unit length in the currently deformed configuration $\left({ }^{2} C\right.$ state) are especially considered here in contrast to be ignored in previous literatures. Finally, nonlinear terms of the virtual work done by boundary moments per unit length in the ${ }^{2} C$ state are also derived. Advantages of this new theory not only come from passing the rigid body rule, which is seldom found in the nonlinear theory of the plate, but also owing to the completeness of the proposed theory.
\end{abstract}

\section{INTRODUCTION}

The incremental virtual work equation is usually encountered in the buckling analysis and mainly formulated in the currently deformed configuration $\left({ }^{2} C\right.$ state). Since the currently deformed configuration is unknown, the Lagrangian for-

Author for correspondence: Shyh-Rong Kuo (e-mail: srkuo@mail.ntou.edu.tw). *Department of Harbor and River Engineering, National Taiwan Ocean University, No. 2, Pei-Ning Road, Keelung 20224, Taiwan, R.O.C.

**Computation and Simulation Center, National Taiwan Ocean University, No. 2, Pei-Ning Road, Keelung 20224, Taiwan, R.O.C.

***Department of Civil Engineering, National Taiwan University, No. 1, Sec. 4, Roosevelt Road, Taipei 10617, Taiwan, R.O.C. mulation is usually adopted to describe the current deformations within the known reference coordinates system. If the reference coordinates system is based on an un-deformed configuration $\left({ }^{0} \mathrm{C}\right.$ state), the formulation is called the total Lagrange formulation; in contrast, if the coordinates system is based on the previously deformed configuration $\left({ }^{1} C\right.$ state), this formulation is called the update Lagrange formulation.

In early studies of the stability theory for plates and shells, a known configuration was first taken as the reference configuration for describing the equilibrium of actions (forces and moments) and thus, forces equilibrium equations in the currently deformed configuration, ${ }^{2} C$ state, could be constructed by using the coordinates system in the reference configuration and by considering incremental displacements effects. Through a relationship between forces and displacements, governing equations of buckling were obtained by using the incremental displacements. For example, the well-known von Kármán formula used the ${ }^{0} C$ state as the reference configuration, and those theories of buckling developed by Timoshenko and Gere [10], Chajes [3] and Ziegler [23] used the ${ }^{1} C$ state as the reference configuration. Only the membrane forces are considered in theories, their governing equations are easy to understand and each term has its own physical meaning. Therefore, they are still in use for the researchers nowadays [9, 13, 16, 22] Although these buckling theories discussed stability behaviors of thin plates; however, only considering the membrane forces effects leads to fail in handling those buckling problems with out-of-plane actions. In the theory developed by Hodges et al. [5], the energy approach was adopted to derive the governing equation for plate buckling. In their theory, warping effect was systematically eliminated, large deformation and rotation were both taken into consideration, and the governing equation was constructed by taking forces and moments as variables. However, implicit type of the govern equation and complexity of each term in the governing equation make it hard to understand and implement.

As known, the principle of virtual work is a very powerful tool to derive the governing equation of mechanics $[1,15]$. Unlike the previously mentioned approaches, the virtual work method only needs to set up the strain energy terms and considers the virtual work done by the actions. By using the total Lagrange formulation or the update Lagrange formulation, one can construct the virtual work in the ${ }^{2} C$ state by way of the 
known configurations $\left({ }^{0} \mathrm{C}\right.$ or ${ }^{1} \mathrm{C}$ state). Introducing the relation of displacements and strains, one can formulate the governing equation and natural boundary conditions from the incremental virtual work equation by means of the variational principle. Yang and Kuo [19] adopted the update Lagrange formulation to develop the nonlinear buckling theory for a solid beam. They used the rigid body motion test to examine the governing equation, natural boundary condition and incremental virtual work equation for validation. Basically, the virtual work method is more systematical than the previously mentioned approaches due to its completeness; however, using the principle of virtual work to study the stability of a plate is seldom found. The possible reasons for this situation may come from two folds: (1) some terms in the virtual strain energy do not have clear physical meanings; (2) the boundary forces and nonlinear virtual work done in the ${ }^{2} C$ state should be defined correctly.

In this paper, the update Lagrange formulation is adopted and the virtual work method is used to derive the incremental virtual work equation for the nonlinear buckling analysis of a thin plate. Three issues in the derivations are crucial: (1) stain energy due to six nonlinear strain components should be all taken into considered; (2) the boundary moments per unit length and the effective transverse edge force per unit length acting on the boundary of the middle surface in the ${ }^{2} C$ state are defined; (3) the nonlinear virtual work done by the boundary moments in the ${ }^{2} C$ state is derived. It should be noted that those terms with unclear physical meanings in the incremental virtual strain equation are cancelled out by some mathematical operations. Besides, based on the rigid body rule $[7,17,18$, 19], a rigid body test especially for a plate to check the incremental virtual work equation is also proposed. In this regard, the proposed theory can satisfy the rigid body test such that the objectivity of the proposed theory is guaranteed. The proposed theory has several merits: (1) the incremental virtual work equation are represented in the explicit forms such that the physical meaning for each term is clear; (2) the theory considers all kinds of actions such that it has a wider application; (3) the theory can pass the rigid body rule such that it is suitable in analyzing the nonlinear behavior in which case large deformation is usually encountered.

\section{STATICS AND KINEMATICS OF A THIN PLATE}

To analyze the stability behaviors of a structure, two stages should be considered. The first one is called the pre-buckling stage in which the deformation after loadings is very small and consequently, the influence due to geometrical changes can be neglected. The second is called the buckling stage in which a small increment in the loading will result in large deformations. In this paper, the update Lagrange formulation is adopted. It means that the previous deformed configuration $\left({ }^{1} C\right.$ state) obtained from the pre-buckling stage is used to derive the incremental equilibrium equation in the currently deformed

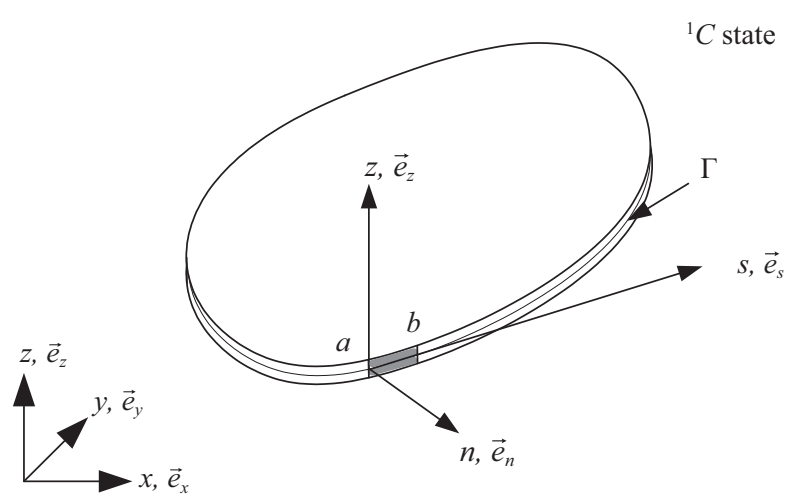

(a) Coordinates of a thin plate in ${ }^{1} C$ state.

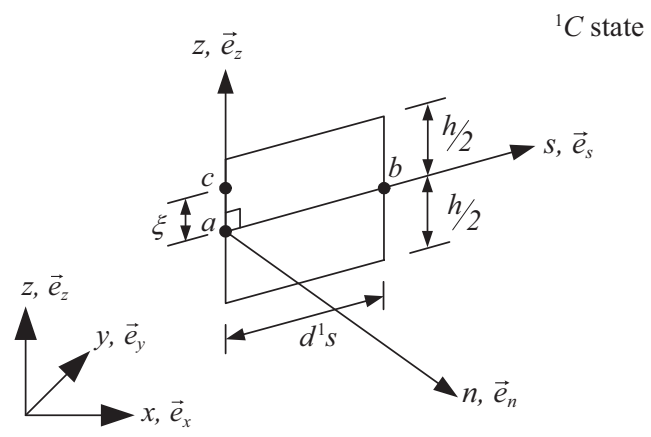

(b) Infinitesimal boundary surface element.

Fig. 1. Plate in ${ }^{1} C$ state.

configuration $\left({ }^{2} C\right.$ state $)$ for the buckling stage. In what following, the pre-buckling and buckling stages are analyzed consequently.

\section{Pre-buckling Stage in ${ }^{1} C$ State}

A thin plate, shown in Fig. 1(a), having a thickness $h$ with its middle surface locating on $x-y$ plane, in which the boundary of the middle surface is denoted as $\Gamma$, is considered. At the edge of this thin plate, a small surface element is chosen as shown in Fig. 1(b). The curve $a b$ is a part of $\Gamma$ and the segment $a c$ is perpendicular to the curve $a b$. Define a local coordinate $n-s-z$ system on this small surface element with the unit vector of $s$ - axis, $\vec{e}_{s}$, denoting the tangential direction of $\Gamma$ which is perpendicular to the $z$ - axis (direction of segment $a b$ ); the unit vector of $n$-axis, $\vec{e}_{n}$, denoting the out-normal direction of this tiny surface element; and the unit vector of $z$ - axis, $\vec{e}_{z}$, denoting the direction of the plate thickness (direction of segment $a c$ ). The length of curve $a b$ is denoted as $d^{1} s$ and the length of segment $a c$ is $\xi$, i.e., the distance from point $c$ to the middle surface.

According to Kirchhoff thin plate theory [14], the linear displacement fields for the thin plate can be expressed as:

$$
\begin{aligned}
& u_{x}=u+\xi \theta_{y}, \\
& u_{y}=v-\xi \theta_{x},
\end{aligned}
$$




$$
\begin{gathered}
u_{z}=w \\
\theta_{y}=-\frac{\partial w}{\partial x} \\
\theta_{x}=\frac{\partial w}{\partial y}
\end{gathered}
$$

in which $u, v$, and $w$ denote the displacements for the middle surface in $x$ -,$y$ - and $z$ - directions, respectively, $u_{x}, u_{y}$ and $u_{z}$ denote the displacements for point $\mathrm{c}$ in $x$-, $y$ - and $z$-directions, respectively, and $\theta_{x}$ and $\theta_{y}$ denote the rotational angles for segment $a c$ with respect to $x$ - axis and $y$ - axis, respectively. Integrating the stress components with respect to the plate thickness, the membrane forces per unit length, transverse shears per unit length and moments per unit length, respectively, can be obtained:

$$
\begin{gathered}
{ }^{1} N_{x x}=\int_{-h / 2}^{h / 2} \tau_{x x} d \xi,{ }^{1} N_{y y}=\int_{-h / 2}^{h / 2} \tau_{y y} d \xi, \\
{ }^{1} N_{x y}=\int_{-h / 2}^{h / 2} \tau_{x y} d \xi, \\
{ }^{1} Q_{x}=\int_{-h / 2}^{h / 2} \tau_{x z} d \xi,{ }^{1} Q_{y}=\int_{-h / 2}^{h / 2} \tau_{y z} d \xi, \\
{ }^{1} M_{x x}=\int_{-h / 2}^{h / 2} \tau_{x x} \xi d \xi,{ }^{1} M_{y y}=\int_{-h / 2}^{h / 2} 1 \tau_{y y} \xi d \xi, \\
{ }^{1} M_{x y}=\int_{-h / 2}^{h / 2} \tau_{x y} \xi d \xi .
\end{gathered}
$$

In the above equations, the index ' 1 ' on the left-upper corner denotes the physical quantity in the ${ }^{1} C$ state, $\tau_{i j}$ is the stress component, $N_{i j}$ is the membrane force per unit length, $Q_{i}$ is the transverse shear per unit length, and $M_{i j}$ is the moment per unit length.

As shown in Fig. 1(b), the transformation relationship between $n-s-z$ local coordinate system and global $x-y-z$ coordinate system in ${ }^{1} C$ state can be expressed as

$$
\left\{\begin{array}{l}
\vec{e}_{n} \\
\vec{e}_{s} \\
\vec{e}_{z}
\end{array}\right\}=\left[\begin{array}{ccc}
n_{x} & n_{y} & 0 \\
-n_{y} & n_{x} & 0 \\
0 & 0 & 1
\end{array}\right]\left\{\begin{array}{l}
\vec{e}_{x} \\
\vec{e}_{y} \\
\vec{e}_{z}
\end{array}\right\},
$$

where $n_{x}$ and $n_{y}$ are $x$ - and $y$ - components for the out-normal vector, $\vec{e}_{n}$, respectively. The rotation angles of the surface element, i.e., the shaded region containing segment $a b$ shown in Fig. 1(b) in the local coordinate system, can be expressed in the following. First, two rotational angles in $s$ - and $n$ - directions are

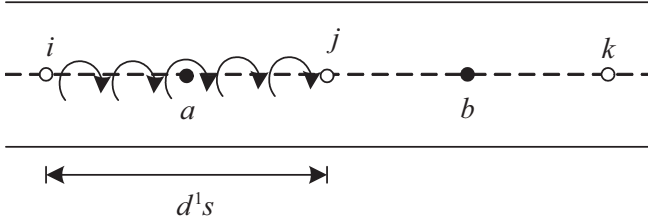

(a) Total twisting moment per unit length for the normal direction in ${ }^{1} C$ state.

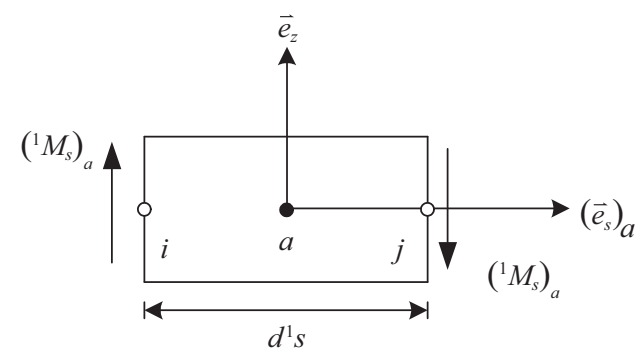

(b) Equivalent force couples on boundary $i j$.

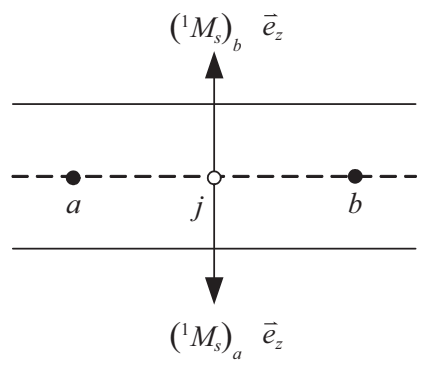

(c) Total force at point $j$.

Fig. 2. Boundary equivalent vertical force in ${ }^{1} C$ state.

$$
\theta_{s}=-\frac{\partial w}{\partial n}, \theta_{n}=\frac{\partial w}{\partial^{1} s}
$$

where $\theta_{s}$ and $\theta_{n}$ represent rotation angles in $s$ - and $n$-directions, respectively; while the rotational angle in $z$-direction, $\theta_{z}^{*}$, is

$$
\theta_{z}^{*}=-n_{x} \frac{\partial u}{\partial^{1} s}-n_{y} \frac{\partial v}{\partial^{1} s} .
$$

The boundary traction force in ${ }^{1} C$ state, $\stackrel{\vec{t}}{t}$, can be written as

$$
\begin{aligned}
{ }^{1 \vec{t}} & ={ }^{1} t_{x} \vec{e}_{x}+{ }^{1} t_{y} \vec{e}_{y}+{ }^{1} t_{z} \vec{e}_{z} \\
& ={ }^{1} t_{n} \vec{e}_{n}+{ }^{1} t_{s} \vec{e}_{s}+{ }^{1} t_{z} \vec{e}_{z} .
\end{aligned}
$$

Since $\vec{e}_{s}$ and $\vec{e}_{n}$ are perpendicular to $\vec{e}_{z}$, respectively, boundary moments per unit length, bending moment ${ }^{1} M_{n}$ and twisting moment ${ }^{1} M_{s}$, acting on the boundary due to the traction components, ${ }^{1} t_{s}$ and ${ }^{1} t_{n}$, can be defined as

$$
{ }^{1} M_{n}=\int_{-h / 2}^{h / 2} t_{n} \xi d \xi,{ }^{1} M_{s}=\int_{-h / 2}^{h / 2}{ }^{1} t_{s} \xi d \xi
$$

As shown in Fig. 2(a), the twisting moment per unit length for the normal direction in ${ }^{1} C$ state at the boundary point $a$ can 
be expressed as $\left({ }^{1} M_{s}\right)_{a}$. Considering an infinitesimal segment $i j$ with length equal to $d^{1} s$ shown in Fig. 2(b), the total twisting moment for the normal direction in ${ }^{1} C$ state then can be written as $\left({ }^{1} M_{s}\right)_{a} d{ }^{1} s$. Following with those works of Timoshenko [11], Ugral [12] and Wempner [14], such a total twisting moment can be replaced by an equivalent force couple, as shown in Fig. 2(b). It means the total twisting moment for the normal direction in ${ }^{1} C$ state acting on this infinitesimal element can be equivalent to a force couple system; that is, $\left({ }^{1} M_{s}\right)_{a} \vec{e}_{z}$ acting at point $i$ and $-\left({ }^{1} M_{s}\right)_{a} \vec{e}_{z}$ acting at point $j$. Now considering a neighbor point $b$ with a distance between two points (point $a$ and point $b$ ) equal to $d^{1} s$ shown in Fig. 2(c), the force acing at point $j$, which is contributed from the equivalent force couple in the infinitesimal element centered at point $b$, is $\left({ }^{1} M_{s}\right)_{b} \vec{e}_{z}$; therefore, the total force at point $j$ can be written as $\left({ }^{1} M_{s}\right)_{b} \vec{e}_{z}-\left({ }^{1} M_{s}\right)_{a} \vec{e}_{z}$ and such a total force acts on a single point. Considering a continuously distributed concept, the equivalent vertical force per unit length, ${ }^{1} \vec{V}$, can be expressed as

$$
{ }^{1} \vec{V}=\frac{1}{d^{1} s}\left[\left({ }^{1} M_{s}\right)_{b} \vec{e}_{z}-\left({ }^{1} M_{s}\right)_{a} \vec{e}_{z}\right] \approx \frac{\partial}{\partial^{1} s}\left({ }^{1} M_{s} \vec{e}_{z}\right) .
$$

In the above equation, it can be found that the twisting moment acting on the boundary of the middle surface, ${ }^{1} M_{s}$, is transformed into a continuously distributed equivalent vertical force per unit length. Therefore, the effective boundary forces per unit length acting on the boundary of the middle surface, $\Gamma$, can be expressed as

$$
\left\{\begin{array}{c}
{ }^{1} N_{x} \\
{ }^{1} N_{y} \\
{ }^{1} N_{z}
\end{array}\right\}=\left(\int^{h / 2} / 2\left\{\begin{array}{c}
{ }^{1} t_{x} \\
{ }^{1} t_{y} \\
{ }^{1} t_{z}
\end{array}\right\} d \xi\right)+\frac{\partial}{\partial^{1} s}\left\{\begin{array}{c}
0 \\
0 \\
{ }^{1} M_{S}
\end{array}\right\} .
$$

When we consider the thin plate problem to be a two-dimensional plane problem as shown in Fig. $3,{ }^{1} N_{x},{ }^{1} N_{y}$ and ${ }^{1} N_{z}$ are $x$ - $y$ - and $z$ - components of the boundary force per unit edge length acting on the boundary $\Gamma$, respectively. It should be note that ${ }^{1} N_{z}$ is the well-known Kirchhoff's force. Since the twisting moment per unit length, ${ }^{1} M_{s}$ has been transformed into the equivalent vertical force per unit length, the boundary actions on $\Gamma$ only contains a boundary bending moment per unit length, ${ }^{1} M_{n}$, and three boundary forces per unit length, ${ }^{1} N_{x},{ }^{1} N_{y}$ and ${ }^{1} N_{z}$. Without considering body forces, the equation of equilibrium in $z$ - direction in ${ }^{1} C$ state can be written as

$$
\frac{\partial^{1} \tau_{x z}}{\partial x}+\frac{\partial^{1} \tau_{y z}}{\partial y}+\frac{\partial^{1} \tau_{z z}}{\partial z}=0
$$

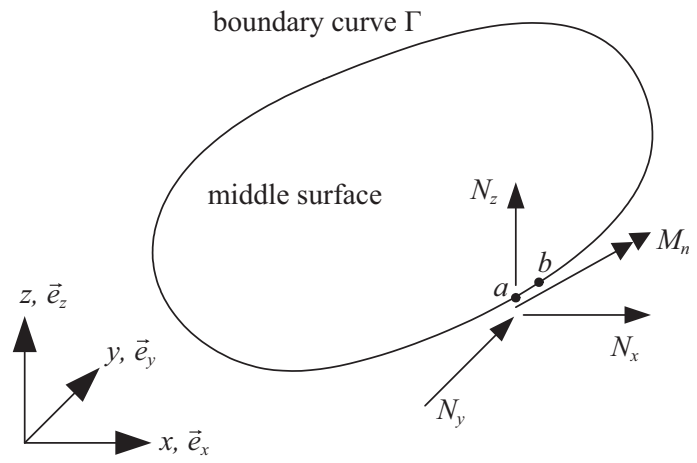

Fig. 3. Illustration of the plane problem for the thin plate.

Multiplying (16) by $z$ and integrating with respect to the plate thickness and using Green's theorem with the traction-free conditions [21] can yield

$$
\begin{aligned}
& \frac{\partial}{\partial x} \int_{-h / 2}^{h / 2} z^{1} \tau_{x z} d z+\frac{\partial}{\partial y} \int_{-h / 2}^{h / 2} z^{1} \tau_{y z} d z \\
& \quad=-\int_{-h / 2}^{h / 2} z \frac{\partial^{1} \tau_{z z}}{\partial z} d z \\
& \quad=\int_{-h / 2}^{h / 2} \tau_{z z} d z .
\end{aligned}
$$

Equation (17) will be used to deal with an unclear term appearing in the following derivation, in which the integration of normal stress components along the direction of plate thickness is ambiguous.

\section{Buckling Stage in ${ }^{2} C$ State}

When the plate deformed form the ${ }^{1} C$ state (previous deformed configuration) to the ${ }^{2} C$ state (currently deformed configuration), the deformation includes displacements $(u, v$, and $w)$ at point $a$ and rotational angles $\left(\theta_{n}, \theta_{s}\right.$, and $\left.\theta_{z}^{*}\right)$ for the small surface element at the edge. Illustration of the surface element deformation in the ${ }^{2} C$ state is shown in Fig. 4, in which points $a^{\prime}, b^{\prime}$ and $c^{\prime}$ represent the current positions of points $a, b$ and $c$ in ${ }^{1} C$ state, respectively, and $d^{2} s$ denotes the length of $a^{\prime} b^{\prime}$. According to the assumptions in the Kirchhoff plate theory, the length of $a^{\prime} c^{\prime}$ remains the same as that of segment $a c$, i.e., $\xi$. Similar to that in the ${ }^{1} C$ state, a coordinate system $\left(\alpha-\beta-\gamma\right.$ system) at the plate edge in the ${ }^{2} C$ state can be defined. The unit vector of $\beta$ axis, $\vec{e}_{\beta}$, is in the tangential direction of the boundary of the middle surface $(\Gamma)$, i.e., in the direction of $a^{\prime} b^{\prime}$. The unit vector of $\gamma$ axis, $\vec{e}_{\gamma}$, is in the direction of the plate thickness, i.e., in the direction of $a^{\prime} c^{\prime}$. The unit vector of $\alpha$ axis, $\vec{e}_{\alpha}$, is in the normal direction of the plate edge.

The unit vectors in the previous deformed configuration $\left({ }^{1} C\right.$ state) and those in the currently deformed configuration $\left({ }^{2} C\right.$ state) have the following transformation: 


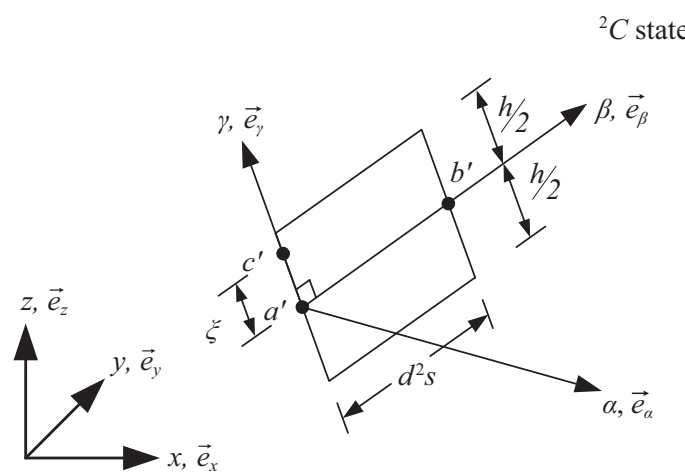

Fig. 4. $(\alpha-\beta-\gamma)$ Coordinates in ${ }^{2} C$ state.

$$
\left\{\begin{array}{l}
e_{\alpha} \\
e_{\beta} \\
e_{\gamma}
\end{array}\right\}=\left[\begin{array}{ccc}
1 & \theta_{z}^{*} & -\theta_{s} \\
-\theta_{z}^{*} & 1 & \theta_{n} \\
\theta_{s} & -\theta_{n} & 1
\end{array}\right]\left\{\begin{array}{l}
e_{n} \\
e_{s} \\
e_{z}
\end{array}\right\}
$$

Define the linear strain components for a boundary point using $n-s-z$ - coordinates as

$$
\begin{gathered}
e_{s s}=-n_{y} \frac{\partial u}{\partial^{1} s}+n_{x} \frac{\partial v}{\partial^{1} s}, \\
e_{n n}=n_{x} \frac{\partial u}{\partial^{1} n}+n_{y} \frac{\partial v}{\partial^{1} n}, \\
e_{s n}=\frac{1}{2}\left(n_{x} \frac{\partial u}{\partial^{1} s}+n_{y} \frac{\partial v}{\partial^{1} s}\right)+\frac{1}{2}\left(-n_{y} \frac{\partial u}{\partial^{1} n}+n_{x} \frac{\partial v}{\partial^{1} n}\right)
\end{gathered}
$$

From (19), the relationship of the infinitesimal length $d^{1} s$ in the ${ }^{1} C$ state and its corresponding deformed infinitesimal length $d^{2} s$ in the ${ }^{2} C$ state can be written as

$$
d^{2} s=d^{1} s\left(1+e_{s s}\right) .
$$

The tractions acting at the plate edge in the ${ }^{2} C$ state, as shown in Fig. 4, can be expressed by the coordinate system in the ${ }^{2} C$ state as well as the coordinate system in the ${ }^{1} C$ state:

$$
\begin{aligned}
{ }^{2 \vec{t}} & ={ }^{2} t_{x} \vec{e}_{x}+{ }^{2} t_{y} \vec{e}_{y}+{ }^{2} t_{z} \vec{e}_{z} \\
& ={ }^{2} t_{n} \vec{e}_{n}+{ }^{2} t_{s} \vec{e}_{s}+{ }^{2} t_{z} \vec{e}_{z} \\
& ={ }^{2} t_{\alpha} \vec{e}_{\alpha}+{ }^{2} t_{\beta} \vec{e}_{\beta}+{ }^{2} t_{\gamma} \vec{e}_{\gamma} .
\end{aligned}
$$

Since $\vec{e}_{\beta}$ and $\vec{e}_{\alpha}$ are both perpendicular to $\vec{e}_{\gamma}$, the boundary moments per unit length due to the tractions in the ${ }^{2} C$ state, ${ }^{2} t_{\beta}$ and ${ }^{2} t_{\alpha}$, can be defined as

$$
{ }^{2} M_{\beta}=\int_{-h / 2}^{h / 2} t_{\beta} \xi d \xi,{ }^{2} M_{\alpha}=\int_{-h / 2}^{h / 2}{ }^{2} t_{\alpha} \xi d \xi
$$

Because the traction component, ${ }^{2} t_{\gamma}$, acts in the direction parallel to the plate thickness after deformation, it results in no moment consequently.

By using (18), two traction components in the $n-s-z$ - coordinate can be obtained as

$$
\begin{aligned}
& { }^{2} t_{n}={ }^{2} t_{\alpha}-{ }^{2} t_{\beta} \theta_{z}^{*}+{ }^{2} t_{\gamma} \theta_{s}, \\
& { }^{2} t_{s}={ }^{2} t_{\alpha} \theta_{z}^{*}+{ }^{2} t_{\beta}-{ }^{2} t_{\gamma} \theta_{n} .
\end{aligned}
$$

Multiplying (25) and (26) by $\xi$, integrating with respect to plate thickness and introducing (24), one can have

$$
\begin{aligned}
& \int_{-h / 2}^{h / 2}{ }^{2} t_{n} \xi d \xi={ }^{2} M_{\alpha}-{ }^{2} M_{\beta} \theta_{z}^{*}+\int_{-h / 2}^{h / 2}{ }^{2} t_{\gamma} d \xi \theta_{s}, \\
& \int_{-h / 2}^{h / 2}{ }^{2} t_{s} \xi d \xi={ }^{2} M_{\alpha} \theta_{z}^{*}+{ }^{2} M_{\beta}-\int_{-h / 2}^{h / 2}{ }^{2} t_{\gamma} \xi \xi \theta_{n} .
\end{aligned}
$$

It should be noted that in the ${ }^{2} C$ state the unit vectors $\vec{e}_{s}$ and $\vec{e}_{n}$ do not perpendicular to $\vec{e}_{\gamma}$. From the fundamental concept of moments, the left-hand side terms appearing in (27) and (28) cannot be taken as the moments per unit length acting on the middle surface, which are done by the tractions in the ${ }^{2} C$ state; therefore, those integration terms appearing in the right-hand sides of (27) and (28) do not show apparent physical meanings.

As shown in Fig. 5(a), the twisting moment per unit length in the normal direction for point $a^{\prime}$ (the ${ }^{2} C$ state) can be expressed by $\left({ }^{2} M_{\beta}\right)_{a}$. Taking an infinitesimal segment $i j$ with length $d^{2} s$ in the neighborhood of point $a^{\prime}$ (shown in Fig. $5(\mathrm{~b})$ ), the total twisting moment acting on segment $i j$ can be written as $\left({ }^{2} M_{\beta}\right)_{a} d^{2} s$, which should be equivalent to a force couple system with a force $-\left({ }^{2} M_{\beta}\right)_{a}\left(\vec{e}_{\gamma}\right)_{a}$ acting at point $j$ and the other force $\left({ }^{2} M_{\beta}\right)_{a}\left(\vec{e}_{\gamma}\right)_{a}$ acting at point $i$. In the above statement, $\left(\vec{e}_{\gamma}\right)_{a}$ represents the unit vector in the direction of plate thickness for point $a^{\prime}$ after deformation (the ${ }^{2} C$ state). Based on the same argument, there exists an equivalent force couple system acting at point $b^{\prime}$, which is a neighbor point of point $a^{\prime}$ and the length of segment $a^{\prime} b^{\prime}$ is $d^{2} s$. As mentioned earlier, this force couple system results in a force, $\left({ }^{2} M_{\beta}\right)_{b}\left(\vec{e}_{\gamma}\right)_{b}$, acting at point $j$, where the vector $\left(\vec{e}_{\gamma}\right)_{b}$ represents the unit vector in the direction of plate thickness for point $b^{\prime}$ after deformation (the ${ }^{2} C$ state) and is not necessary 


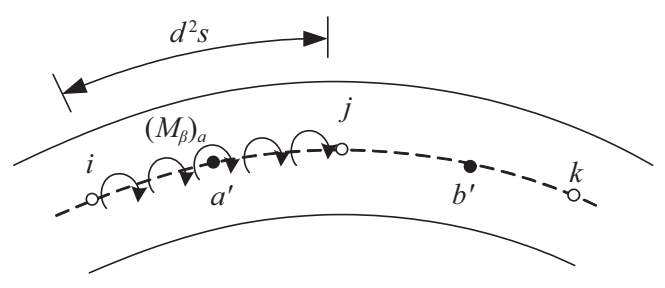

(a) Total twisting moment per unit length for the normal direction in ${ }^{2} \mathrm{C}$ state.

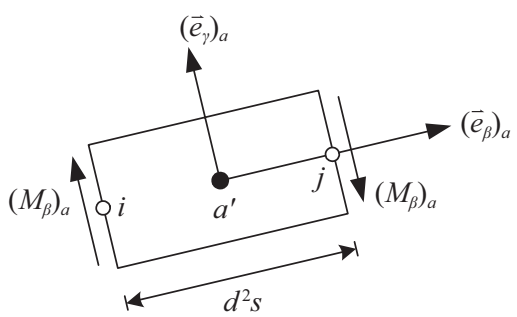

(b) Equivalent force couples on boundary ij.

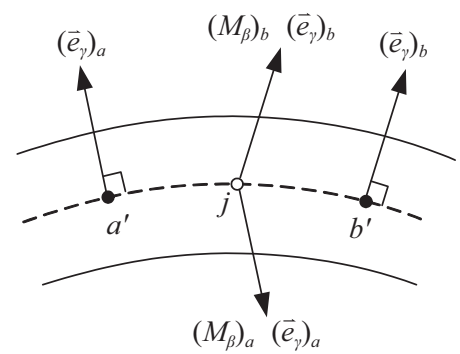

(c) Total force at point $j$.

Fig. 5. Boundary equivalent vertical force in ${ }^{2} C$ state.

equal to $\left(\vec{e}_{\gamma}\right)_{a}$. As can be seen in Fig. 5(c), the total equivalent vertical force at point $j$ is equal to $\left({ }^{2} M_{\beta}\right)_{b}\left(\vec{e}_{\gamma}\right)_{b}-\left({ }^{2} M_{\beta}\right)_{a}$ $\left(\vec{e}_{\gamma}\right)_{a}$. Considering continuous distribution of such an equivalent force, the equivalent vertical force per unit length in the ${ }^{2} C$ state, ${ }^{2} \vec{V}$, can be expressed as

$$
\begin{aligned}
{ }^{2} \vec{V} & =\frac{1}{d^{2} s}\left({ }^{2} M_{\beta}\right)_{b}\left(\vec{e}_{\gamma}\right)_{b}-\left({ }^{2} M_{\beta}\right)_{a}\left(\vec{e}_{\gamma}\right)_{a} \\
& \approx \frac{\partial}{\partial^{2} s}\left({ }^{2} M_{\beta} \vec{e}_{\gamma}\right) .
\end{aligned}
$$

From (9) and (19), one has

$$
\vec{e}_{\gamma}=\theta_{y} \vec{e}_{x}-\theta_{x} \vec{e}_{y}+\vec{e}_{z}
$$

With the use of (30), Eq. (29) can rewritten as

$$
{ }^{2} \vec{V}=\frac{\partial}{\partial^{2} s}\left({ }^{2} M_{\beta} \theta_{y} \vec{e}_{x}-{ }^{2} M_{\beta} \theta_{x} \vec{e}_{y}+{ }^{2} M_{\beta} \vec{e}_{z}\right) .
$$

Equation (31) transforms the twisting moment per unit length ${ }^{2} M_{\beta}$ into equivalent force per unit length. Similarly, the effective boundary forces per unit length acting on the boundary of the middle surface $\Gamma$ in the ${ }^{2} C$ state can be yielded as:

$$
\left\{\begin{array}{c}
{ }^{2} N_{x} \\
{ }^{2} N_{y} \\
{ }^{2} N_{z}
\end{array}\right\}=\left(\int_{-h / 2}^{h / 2}\left\{\begin{array}{c}
{ }^{2} t_{x} \\
{ }^{2} t_{y} \\
{ }^{2} t_{z}
\end{array}\right\} d \xi\right)+\frac{\partial^{2}}{\partial^{2} s}\left\{\begin{array}{c}
{ }^{2} M_{\beta} \theta_{y} \\
-{ }^{2} M_{\beta} \theta_{x} \\
{ }^{2} M_{\beta}
\end{array}\right\} .
$$

In the above equation, ${ }^{2} N_{x},{ }^{2} N_{y}$ and ${ }^{2} N_{z}$ are boundary forces per unit length acting on $\Gamma$ in the ${ }^{2} C$ state and they can be considered as a generalized Kirchhoff's force. These effective forces per unit length in the ${ }^{2} C$ state are firstly defined to authors' best knowledge. They are clearly defined only when the deformed positions in the ${ }^{2} C$ state are clearly handled.

\section{INCREMENTAL VIRTUAL WORK EQUATION}

In this section, the update Lagrangian formulation will be used to derive the incremental virtual work equation. The incremental virtual work equation is written as $[1,19]$ :

$$
\int_{{ }^{1} V} C_{k l i j} e_{k l} \delta e_{i j} \quad d^{1} V+\int_{{ }^{1} V}^{1} \tau_{i j} \delta \eta_{i j} \quad d^{1} V={ }^{2} R-{ }^{1} R,
$$

where

$$
{ }^{2} R=\oint_{{ }_{2}}^{2} t_{i} \delta u_{j} d^{2} S, i=x, y, z,
$$

and

$$
{ }^{1} R=\oint_{S}^{1} t_{i} \delta u_{j} d^{1} S, i=x, y, z
$$

In the above equations, ${ }^{2} R$ and ${ }^{1} R$ represent the virtual work done by the traction forces in ${ }^{2} C$ and ${ }^{1} C$ states, respectively; $u_{i}$ is the incremental displacement from the ${ }^{1} C$ state to the ${ }^{2} C$ state; ${ }^{1} S$ and ${ }^{2} S$ represent the surface enclosed the plate in the ${ }^{1} C$ state and ${ }^{2} C$ state, respectively; ${ }^{1} t_{i}$ and ${ }^{2} t_{i}$ are traction forces action on surface ${ }^{1} S$ and ${ }^{2} S$ in the ${ }^{1} C$ state and ${ }^{2} C$ state, respectively.

The first and second terms in the left hand side of (33) is the linear virtual strain energy, $\delta U_{E}$, and nonlinear incremental virtual strain energy, $\delta U_{N}$, respectively. That is

$$
\begin{gathered}
\delta U_{E}=\int_{{ }^{\mathrm{I}} V} C_{k l i j} e_{k l} \delta e_{i j} d^{1} V^{\prime} \\
\delta U_{N}=\int_{\mathrm{I}_{V}}{ }^{1} \tau_{i j} \delta \eta_{i j} d^{1} V^{\cdot \cdot}
\end{gathered}
$$


in which $C_{i j k l}$ is the elastic modulus tensor, $e_{i j}$ is the linear strain tensor, $\eta_{i j}$ is the nonlinear strain tensor, ${ }^{1} \tau_{i j}$ is the Cauchy stress tensor in the ${ }^{1} C$ state, and ${ }^{1} V$ is the volume in the ${ }^{1} C$ state. Detailed descriptions of the above physical quantities can be found in [19]. It should be noted that ${ }^{2} R$ in (34) is constructed in the currently deformed configuration, i.e., the ${ }^{2} C$ state. In what following, we will derive these terms step by step.

\section{Linear Virtual Strain Energy and Nonlinear Virtual Strain Energy}

Assume the plate is an isotropic linearly elastic material with the Young's modulus $E$ and the Poisson's ratio $v$. Substituting (1) to (5) into (36) yields

$$
\begin{array}{r}
\delta U_{E}=\frac{E h}{2\left(1-v^{2}\right)} \iint\left\{\delta\left(\frac{\partial u}{\partial x}+\frac{\partial v}{\partial y}\right)^{2}\right. \\
\left.+2(1-v) \delta\left[\frac{1}{4}\left(\frac{\partial u}{\partial u}+\frac{\partial v}{\partial x}\right)^{2}-\frac{\partial u}{\partial x} \frac{\partial v}{\partial y}\right]\right\} d A \\
+\frac{E h^{3}}{24\left(1-v^{2}\right)} \int\left\{\delta \delta\left(\frac{\partial^{2} w}{\partial x^{2}}+\frac{\partial^{2} w}{\partial y^{2}}\right)^{2}\right. \\
\left.+2(1-v) \delta\left[\left(\frac{\partial^{2} w}{\partial x \partial y}\right)^{2}-\left(\frac{\partial^{2} w}{\partial x^{2}} \frac{\partial^{2} w}{\partial y^{2}}\right)\right]\right\} d A,
\end{array}
$$

where $d A$ is the surface element of the middle surface. It should be noted that the mentioned linear virtual strain energy is the same as that described in $[10,11]$.

To obtain the nonlinear virtual strain energy, we first substitute (1) to (5) into the nonlinear strain, $\eta_{i j}$, in (37). Using definitions of the membrane force per unit length, moments per unit length and transverse shear force per unit length in (6) to (8), one can obtain the nonlinear virtual strain energy, $\delta U_{N}$, as

$$
\delta U_{N}=\delta U_{G}+\delta U_{c}
$$

in which

$$
\begin{aligned}
\delta U_{G}=\frac{1}{2} \iint & \left\{{ }^{1} N_{x x} \delta\left[\left(\frac{\partial u}{\partial x}\right)^{2}+\left(\frac{\partial v}{\partial x}\right)^{2}+\left(\frac{\partial w}{\partial x}\right)^{2}\right]\right. \\
& +{ }^{1} N_{y y} \delta\left[\left(\frac{\partial u}{\partial y}\right)^{2}+\left(\frac{\partial v}{\partial y}\right)^{2}+\left(\frac{\partial w}{\partial y}\right)^{2}\right] \\
& \left.+2{ }^{1} N_{x y} \delta\left[\frac{\partial u}{\partial x} \frac{\partial u}{\partial y}+\frac{\partial v}{\partial x} \frac{\partial v}{\partial y}+\frac{\partial w}{\partial x} \frac{\partial w}{\partial y}\right]\right\} d A
\end{aligned}
$$

$$
\begin{array}{r}
-\iint\left\{{ }^{1} M_{x x} \delta\left[\frac{\partial u}{\partial x} \frac{\partial^{2} w}{\partial x^{2}}+\frac{\partial v}{\partial x} \frac{\partial^{2} w}{\partial x \partial y}\right]\right. \\
+{ }^{1} M_{y y} \delta\left[\frac{\partial u}{\partial y} \frac{\partial^{2} w}{\partial x \partial y}+\frac{\partial v}{\partial y} \frac{\partial^{2} w}{\partial y^{2}}\right] \\
+{ }^{1} M_{x y} \delta\left[\frac{\partial u}{\partial x} \frac{\partial^{2} w}{\partial x \partial y}+\frac{\partial u}{\partial y} \frac{\partial^{2} w}{\partial x^{2}}\right. \\
\left.+\frac{\partial v}{\partial x} \frac{\partial^{2} w}{\partial y^{2}}+\frac{\partial v}{\partial y} \frac{\partial^{2} w}{\partial x \partial y}\right] \\
-{ }^{1} Q_{x} \delta\left(\frac{\partial u}{\partial x} \frac{\partial w}{\partial x}+\frac{\partial v}{\partial x} \frac{\partial w}{\partial y}\right) \\
\left.-{ }^{1} Q_{y} \delta\left(\frac{\partial u}{\partial y} \frac{\partial w}{\partial x}+\frac{\partial v}{\partial y} \frac{\partial w}{\partial y}\right)\right\} d A
\end{array}
$$

and

$$
\begin{aligned}
\delta U_{c} & =\frac{1}{2} \int\left(\int_{-h / 2}^{h / 2} \tau_{x z} z d z\right) \frac{\partial}{\partial x} \delta\left[\left(\frac{\partial w}{\partial x}\right)^{2}+\left(\frac{\partial w}{\partial y}\right)^{2}\right] d A \\
& +\frac{1}{2} \int\left(\int_{-h / 2}^{h / 2} \tau_{y z} z d z\right) \frac{\partial}{\partial y} \delta\left[\left(\frac{\partial w}{\partial x}\right)^{2}+\left(\frac{\partial w}{\partial y}\right)^{2}\right] d A \\
& +\frac{1}{2} \int\left(\int_{-h / 2}^{h / 2} \tau_{z z} d z\right) \delta\left[\left(\frac{\partial w}{\partial x}\right)^{2}+\left(\frac{\partial w}{\partial y}\right)^{2}\right] d A
\end{aligned}
$$

In $\delta U_{G}$, the nonlinear virtual strain energy contains two parts: one is contributed by three membrane forces per unit length $\left(N_{x x}, N_{y y}\right.$ and $\left.N_{x y}\right)$; the other is contributed by three moments per unit length $\left(M_{x x}, M_{y y}\right.$ and $\left.M_{x y}\right)$ and two transverse shear forces per unit length $\left(Q_{x}\right.$ and $\left.Q_{y}\right)$. The first part had been derived and used by many researchers [3, 10, 23].

In (41), the integrations of stress components in z-direction with respect to the plate thickness cannot show a clear physical meaning. Besides, this virtual strain energy $\delta U_{c}$ has not been discussed in literatures to our best knowledge. Using (17) and Green's theorem, this term can be transformed into

$$
\begin{aligned}
\delta U_{c} & =\frac{1}{2} \oint_{s_{s}}\left[\int_{-h / 2}^{h / 2} z\left({ }^{1} \tau_{x z} n_{x}+{ }^{1} \tau_{y z} n_{y}\right) d z\right] \delta\left[\left(\frac{\partial w}{\partial x}\right)^{2}+\left(\frac{\partial w}{\partial y}\right)^{2}\right] d^{1} s \\
& =\oint_{1_{s}}\left(\int_{-h / 2}^{h / 2} t_{z} z d z\right)\left(\theta_{\mathrm{x}} \delta \theta_{\mathrm{x}}+\theta_{\mathrm{y}} \delta \theta_{\mathrm{y}}\right) d^{1} \mathrm{~s}
\end{aligned}
$$

After transformation, there still exists integration (the integration of the product of traction and $\mathrm{z}$ with respect to the plate thickness) having no definite physical meaning; however, this term will be treated in the following. 


\section{The Virtual Work Done by Tractions in the ${ }^{1} C$ and ${ }^{2} C$ States}

Substituting (1) to (5), (13) and (15) into (35) can yield the virtual work done by the traction force in the ${ }^{1} C$ state as

$$
\begin{aligned}
{ }^{1} R & =\delta W_{1} \\
& =\oint_{1_{s}}\left[{ }^{1} N_{x} \delta u+{ }^{1} N_{y} \delta v+{ }^{1} N_{z} \delta w+{ }^{1} M_{n} \delta \theta_{s}\right] d^{1} s
\end{aligned}
$$

where $\delta W_{1}$ denotes the virtual work done by the effective boundary forces and boundary bending moment in the ${ }^{1} C$ state and is equal to the virtual work done by the traction force in the ${ }^{1} C$ state, i.e., ${ }^{1} R$. Substituting (1) to (5) into (34) , the virtual work done by the traction force in the ${ }^{2} C$ state, i.e., ${ }^{2} R$, can be written as

$$
\begin{aligned}
{ }^{2} R & =\oint_{2_{s}}\left(\int_{-h / 2}^{h / 2}\left\{\begin{array}{c}
{ }^{2} t_{x} \\
{ }^{2} t_{y} \\
{ }^{2} t_{z}
\end{array}\right\}^{T}\left\{\begin{array}{c}
\delta u+\xi \delta \theta_{y} \\
\delta v-\xi \delta \theta_{x} \\
\delta w
\end{array}\right\} d \xi\right) d^{2} s \\
& =\oint_{2_{s}}\left(\int_{-h / 2}^{h / 2}\left\{\begin{array}{c}
{ }^{2} t_{x} \\
{ }^{2} t_{y} \\
{ }^{2} t_{z}
\end{array}\right\}^{T} d \xi\right)\left\{\begin{array}{l}
\delta u \\
\delta v \\
\delta w
\end{array}\right\} d^{2} s \\
& +\oint_{2_{s}}\left(\int_{-h / 2}^{h / 2}\left\{\begin{array}{c}
2 \\
{ }^{2} t_{n} \\
{ }^{2} t_{s}
\end{array}\right\}^{T} \xi d \xi\right)\left\{\begin{array}{c}
\delta \theta_{s} \\
-\delta \theta_{n}
\end{array}\right\} d^{2} s .
\end{aligned}
$$

Substituting (32) into the first integration term in the right hand side of (44), performing integrations by part, and performing coordinate transformation (see Appendix 1) can yield

$$
\begin{aligned}
& \oint_{2_{s}}\left(\int_{-h / 2}^{h / 2}\left\{\begin{array}{c}
{ }^{2} t_{x} \\
{ }^{2} t_{y} \\
{ }^{2} t_{z}
\end{array}\right\}^{T} d \xi\right)\left\{\begin{array}{l}
\delta u \\
\delta v \\
\delta w
\end{array}\right\} d^{2} s \\
& =\oint_{2_{s}}\left[{ }^{2} N_{x} \delta u+{ }^{2} N_{y} \delta v+{ }^{2} N_{z} \delta w+{ }^{2} M_{\beta} \delta \theta_{n}\right] d^{2} s \\
& \quad-\oint_{1_{s}}\left[{ }^{1} M_{s}\left(e_{s s} \delta \theta_{n}+\theta_{n} \delta e_{s s}+\theta_{s} \delta \theta_{z}^{*}\right)\right] d^{1} s .
\end{aligned}
$$

Substituting (27) and (28) into the second integration term in the right hand side of (44), performing coordinate transformation, and neglecting higher order terms (see Appendix 2) can yield

$$
\begin{aligned}
& \oint_{2_{s}}\left(\int_{-h / 2}^{h / 2}\left\{\begin{array}{c}
{ }^{2} t_{n} \\
{ }^{2} t_{s}
\end{array}\right\}^{T} \xi d \xi\right)\left\{\begin{array}{c}
\delta \theta_{s} \\
-\delta \theta_{n}
\end{array}\right\} d^{2} s \\
& =\oint_{2_{s}}\left({ }^{2} M_{\alpha} \delta \theta_{s}-{ }^{2} M_{\beta} \delta \theta_{n}\right) d^{2} s \\
& -\oint_{1_{s}}\left(M_{n} \theta_{z}^{*} \delta \theta_{n}+{ }^{1} M_{s} \theta_{z}^{*} \delta \theta_{s}\right) d^{1} s \\
& +\oint_{1_{s}}\left(\int_{-h / 2}^{h / 2} t_{z} z d z\right)\left(\theta_{x} \delta \theta_{x}+\theta_{y} \delta \theta_{y}\right) d^{1} \mathrm{~s} .
\end{aligned}
$$

Therefore, the virtual work done by the traction force in the ${ }^{2} \mathrm{C}$ state can be obtained by summing up (45) and (46) as:

$$
{ }^{2} R=\delta W_{2}+\delta U_{R}+\delta \hat{U}_{c}
$$

where

$$
\begin{gathered}
\delta W_{2}=\oint_{2_{s}}\left[{ }^{2} N_{x} \delta u+{ }^{2} N_{y} \delta v+{ }^{2} N_{z} \delta w+{ }^{2} M_{\alpha} \delta \theta_{s}\right] d^{2} s \\
\delta U_{R}=-\oint_{1_{s}}^{1} M_{s} \delta\left(e_{s s} \theta_{n}+\theta_{z}^{*} \theta_{s}\right) d^{1} s-\oint_{1_{s}}^{1} M_{n} \theta_{z}^{*} \delta \theta_{n} d^{1} s \\
\delta \hat{U}_{c}=\oint_{1_{s}}\left(\int_{-h / 2}^{h / 2} t_{z} z d z\right)\left(\theta_{\mathrm{x}} \delta \theta_{\mathrm{x}}+\theta_{\mathrm{y}} \delta \theta_{\mathrm{y}}\right) d^{1} s
\end{gathered}
$$

in which $\delta W_{2}$ is the linear virtual work done by the effective boundary forces and boundary moment in the ${ }^{2} C$ state. Traditionally, ${ }^{2} R$ is thought to be equal to $\delta W_{2}$ possibly by the intuition. However, from (47), ${ }^{2} R$ is not equal to $\delta W_{2}$. A similar result has been reported in the work of Yang and Kuo [19] in which they derived the nonlinear stability theory of a solid beam. In (47), $\delta U_{R}$ is the nonlinear virtual work done by the boundary moments after rotations. This term has never been mentioned before. It can be found that $\delta \hat{U}_{c}$ in (50) is the same as $\delta U_{c}$ in (42) and thus, they can be canceled out each other. Therefore, the problem of their physical meaning becomes meaningless. Substituting (38), (39), (43) and (47) into (33), we can obtain the incremental virtual work equation for the Kirchhoff thin plate as

$$
\delta U_{E}+\delta U_{G}-\delta U_{R}=\delta W_{2}-\delta W_{1}
$$

It should be noted that $\delta U_{R}$ is derived based on three procedures: first, completely considering the nonlinear strain energy due to six nonlinear strains; second, reasonably defining the boundary moments per unit length and the equivalent transverse edge forces per unit length in the ${ }^{2} C$ state; the last, deriving the nonlinear virtual work done by the boundary moments per unit length in the ${ }^{2} C$ state. One of the virtual works in (51), $\delta U_{R}$, is in the same order as the traditional nonlinear virtual strain energy, $\delta U_{G}$ and thus, it cannot be 
neglected. In the following, we will prove that neglecting this term will result in failure in passing the rigid body test.

\section{THE RIGID BODY TEST FOR THE INCREMENTAL VIRTUAL WORK EQUATION}

The rigid body test is basically originated from the principle of objectivity in mechanical analysis. The incremental virtual equation, natural boundary condition, and geometric stiffness matrix in geometrically nonlinear analysis of shells and plates all should satisfy this rigid body test. In the early literatures, the rigid body test was usually applied on the linear unloading system at initial state and to check the elastic stiffness matrix derived by using the finite element method for validating its convergence. Assume the beam with actions is initially in equilibrium, Yang and Chiou [18] proposed the rigid body rule for the nonlinear element. They showed that when the finite element is subjected to the rigid body motion, the force acting on the body should rotate and translate according to the rigid body motion but the magnitude of the force remains the same. In their study, the rigid body rule was used to examine the elastic and geometric stiffness matrices. In fact, this rigid body rule can be considered as an extension of the principle of objectivity and is adopted to check the theory of stability.

In this subsection, the rigid body rule is adopted to examine the validity of previously derived incremental virtual work equation. The rigid body motion results in no deformation in the thin plate, it can then be said that $d^{1} s=d^{2} s$. Taking the rigid body rotation with respect to $z$ - axis, $\theta_{z r}$, as an example, we will examine the nonlinear incremental virtual work in (51). As shown in Fig. 3, let us take an infinitesimal length $(a b)$ at the edge $\Gamma$. When the rotation, $\theta_{z r}$, occurs, the effective boundary forces per unit length will rotate as well (see Fig. 6(a)). As a result, the forces acting on $\Gamma$ in the ${ }^{2} C$ state can be expressed as:

$$
\begin{gathered}
{ }^{2} N_{x}={ }^{1} N_{x}-{ }^{1} N_{y} \theta_{z r}, \\
{ }^{2} N_{y}={ }^{1} N_{y}+{ }^{1} N_{x} \theta_{z r}, \\
{ }^{2} N_{z}={ }^{1} N_{z}, \\
{ }^{2} M_{\alpha}={ }^{1} M_{n} .
\end{gathered}
$$

The incremental displacements in $x$-, $y$-and $z$ - axis $\left(u_{r}, v_{r}\right.$ and $w_{r}$ ) due to the rotation are

$$
\begin{gathered}
u_{r}=-y \theta_{z r}, \\
v_{r}=x \theta_{z r}, \\
w_{r}=0 .
\end{gathered}
$$

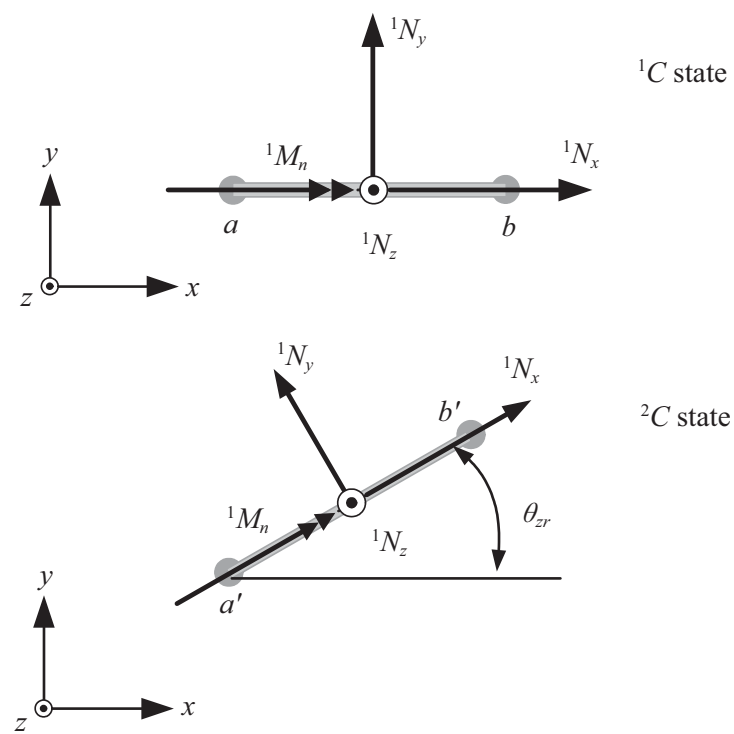

(a) Boundary forces before the rigid body rotation.

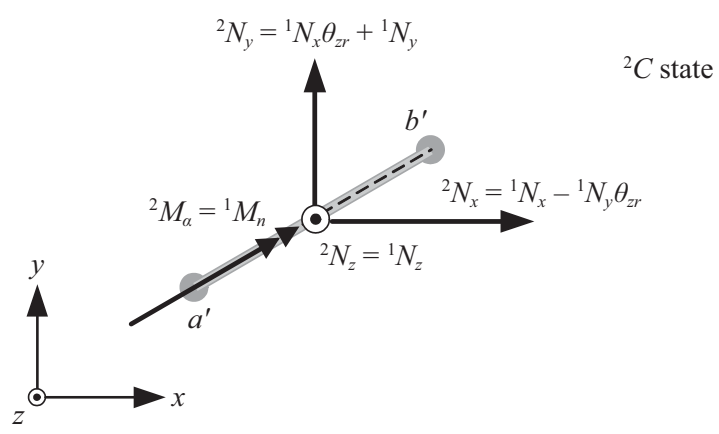

(b) Boundary forces after the rigid body rotation.

Fig. 6. Rigid body rotation $\theta_{z r}$.

Similarly, we consider another two rigid body rotations with respect to $x$ - and $y$-axis, i.e., $\theta_{x r}$ and $\theta_{y r}$, and three rigid body translations with respect to $x$ -,$y$ - and $z$ - axis, i.e., $\Delta_{x r}, \Delta_{y r}$ and $\Delta_{z r}$. The forces and incremental displacements after rigid body motion in the ${ }^{2} C$ state can be expressed as

$$
\begin{gathered}
{ }^{2} N_{x r}={ }^{1} N_{x}-{ }^{1} N_{y} \theta_{z r}+{ }^{1} N_{z} \theta_{y r}, \\
{ }^{2} N_{y r}={ }^{1} N_{z} \theta_{y r}+{ }^{1} N_{y}-{ }^{1} N_{z} \theta_{x r}, \\
{ }^{2} N_{z r}=-{ }^{1} N_{x} \theta_{y r}+{ }^{1} N_{y} \theta_{x r}+{ }^{1} N_{z}, \\
{ }^{2} M_{x r}={ }^{1} M_{n}, \\
u_{r}=\Delta_{x r}-y \theta_{z r}, \\
v_{r}=\Delta_{y r}+x \theta_{z r}, \\
w_{r}=\Delta_{z r}+y \theta_{x r}-x \theta_{y r} .
\end{gathered}
$$


Substituting (63) to (65) into (38), (40) and (49) can yield

$$
\begin{aligned}
& \delta \bar{U}_{E}=0, \\
& \delta \bar{U}_{G}=\theta_{x r} \iint\left({ }^{1} N_{y y} \delta \frac{\partial w}{\partial y}+{ }^{1} N_{x y} \delta \frac{\partial w}{\partial x}\right. \\
& \left.-{ }^{1} Q_{x} \delta \frac{\partial v}{\partial x}-{ }^{1} Q_{y} \delta \frac{\partial v}{\partial y}\right) d A \\
& -\theta_{y r} \iint\left({ }^{1} N_{x x} \delta \frac{\partial w}{\partial x}+{ }^{1} N_{x y} \delta \frac{\partial w}{\partial y}\right. \\
& \left.-{ }^{1} Q_{x} \delta \frac{\partial u}{\partial x}-{ }^{1} Q_{y} \delta \frac{\partial u}{\partial y}\right) d A \\
& +\theta_{z r} \iint\left({ }^{1} N_{x x} \delta \frac{\partial v}{\partial x}-{ }^{1} N_{y y} \delta \frac{\partial u}{\partial y}\right. \\
& \left.-{ }^{1} N_{x y} \delta \frac{\partial u}{\partial x}+{ }^{1} N_{x y} \delta \frac{\partial v}{\partial y}\right) d A \\
& -\theta_{z r} \iint\left({ }^{1} M_{x x} \delta \frac{\partial^{2} w}{\partial x \partial y}-{ }^{1} M_{y y} \delta \frac{\partial^{2} w}{\partial x \partial y}\right. \\
& \left.-{ }^{1} M_{x y} \delta \frac{\partial^{2} w}{\partial x^{2}}+{ }^{1} M_{x y} \delta \frac{\partial^{2} w}{\partial y^{2}}\right) d A \\
& -\theta_{z r} \iint{ }^{1} Q_{x} \delta \frac{\partial w}{\partial y}-{ }^{1} Q_{y} \delta \frac{\partial w}{\partial x} d A, \\
& \delta \bar{U}_{R}=-\theta_{x r} \oint\left[{ }^{1} M_{s}\left(\delta \frac{\partial u_{s}}{\partial s} n_{x}+\delta \frac{\partial u_{n}}{\partial s} n_{y}\right)\right] d s \\
& -\theta_{y r} \oint\left[{ }^{1} M_{s}\left(\delta \frac{\partial u_{s}}{\partial s} n_{y}-\delta \frac{\partial u_{n}}{\partial s} n_{x}\right)\right] d s \\
& +\theta_{z r} \oint\left[{ }^{1} M_{s} \delta \frac{\partial w}{\partial n}-{ }^{1} M_{n} \delta \frac{\partial w}{\partial s}\right] d s .
\end{aligned}
$$

Applying algebraic operation to (66) to (68) and performing Green's theorem with integration by parts, the virtual strain energy in the left hand side of (51) can be write as

$$
\begin{aligned}
\delta \bar{U}_{E}+\delta \bar{U}_{G}-\delta \bar{U}_{R} & =\oint\left({ }^{1} N_{z} \theta_{y r}-{ }^{1} N_{y} \theta_{z r}\right) \delta u d s \\
& +\oint\left({ }^{1} N_{x} \theta_{z r}-{ }^{1} N_{z} \theta_{x r}\right) \delta v d s \\
& +\oint\left({ }^{1} N_{y} \theta_{x r}-{ }^{1} N_{x} \theta_{y r}\right) \delta w d s .
\end{aligned}
$$

Substituting the forces after the rigid body rotation ((59) to (62)) into (48) yields

$$
\begin{aligned}
\delta \bar{W}_{2}=\delta W_{1} & +\oint\left({ }^{1} N_{z} \theta_{y r}-{ }^{1} N_{y} \theta_{z r}\right) \delta u d s \\
& +\oint\left({ }^{1} N_{x} \theta_{z r}-{ }^{1} N_{z} \theta_{x r}\right) \delta v d s \\
& +\oint\left({ }^{1} N_{y} \theta_{x r}-{ }^{1} N_{x} \theta_{y r}\right) \delta w d s
\end{aligned}
$$

Substituting (70) into left hand side of (51), the incremental virtual work can be write as

$$
\begin{aligned}
\delta \bar{W}_{2}-\delta W_{1} & =\oint\left({ }^{1} N_{z} \theta_{y r}-{ }^{1} N_{y} \theta_{z r}\right) \delta u d s \\
& +\oint\left({ }^{1} N_{x} \theta_{z r}-{ }^{1} N_{z} \theta_{x r}\right) \delta v d s \\
& +\oint\left({ }^{1} N_{y} \theta_{x r}-{ }^{1} N_{x} \theta_{y r}\right) \delta w d s
\end{aligned}
$$

Substituting (69) and (71) into (51), one can have

$$
\delta \bar{U}_{E}+\delta \bar{U}_{G}-\delta \bar{U}_{R}=\delta \bar{W}_{2}-\delta W_{1}
$$

which is the same as (51). It means the proposed equation can pass the rigid body test. From the above derivations, it can be concluded that neglecting $\delta U_{R}$ in (51), the incremental virtual work equation cannot pass the rigid body test.

\section{NUMERICAL EXAMPLES}

In what followings, two numerical examples will be provided to investigate the geometrical nonlinear behavior of the shell for validating the proposed theorem and to compare their nonlinear behaviors with the conventional theorem without passing the rigid body rule. In this article, the generalized displacement control method with advantages of efficiency and reliability $[8,20]$, which can be used to pass the limit point and snap-back point steadily in the equilibrium path, is adopted to conduct the incremental iteration analysis. The adopted triangular plate element [7, 17] has three nodes and each of them contains three translation and three rotation degrees of freedom. Besides, for a geometrically nonlinear incrementaliteration analysis, tangential stiffness matrices can be decomposed into two parts: elastic stiffness and geometric stiffness one. To construct the former matrix, a combined use of the plane hybrid element for membrane actions [4] and the HSM element for bending actions [2] is adopted for calculation; while for the latter one three different geometric stiffness matrices are adopted to compare those differences of the proposed theorem and the conventional one. The first stiffness matrix is obtained by using virtual strain energy of (40) and (49) and a combined use of these two equations can pass through the rigid body rule. The second one is the conventional geometric stiffness matrix, which is derived by only considering the nonlinear strain energy in (40). The third one is a simplified geometric stiffness matrix, which is obtained by using the first integrating term in the right-hand side 


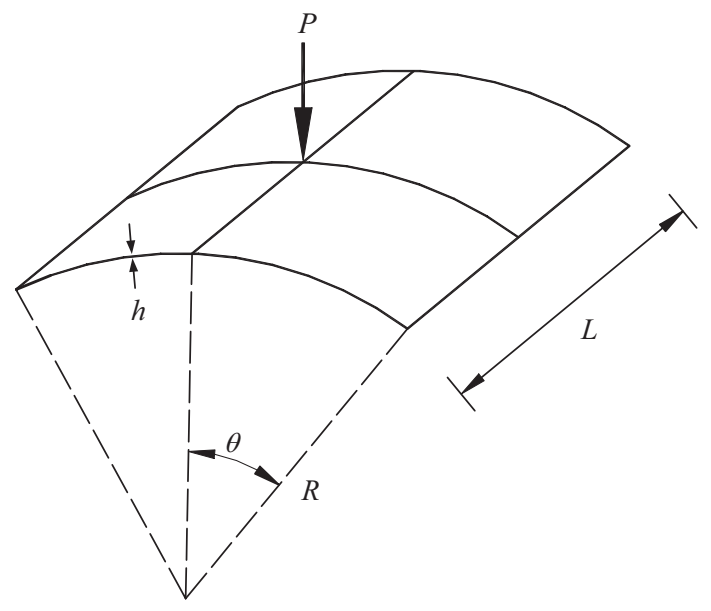

Fig. 7. Cylindrical shell (a concentrated load at the symmetric center).

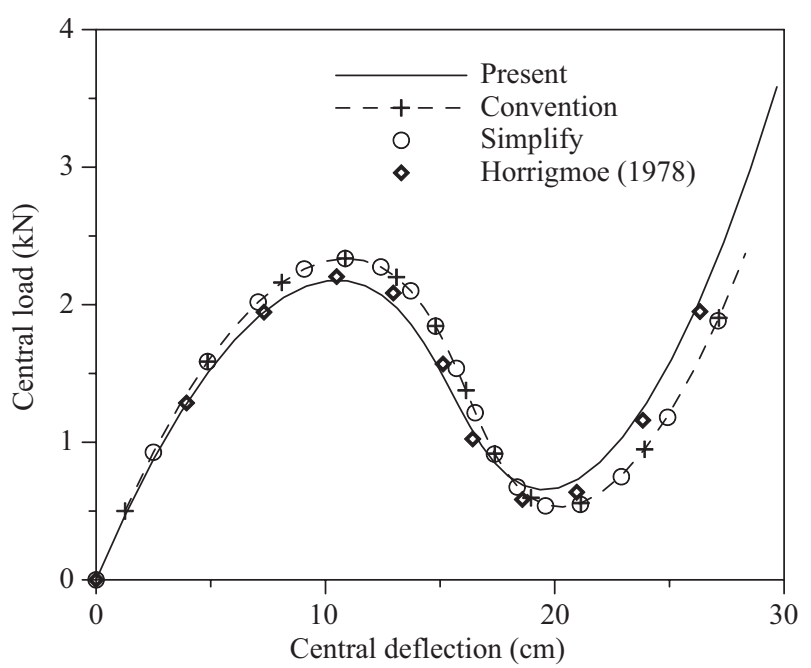

Fig. 8. Central deflection of cylindrical shell.

of (40) and only considering the geometrically nonlinear effect induced by the membrane force of the plate.

\section{Example 1:}

A circular cylindrical shell subjected to a concentrated load $P$ at the geometric center with two straight-line boundaries hinged and curved boundaries free-supported shown in Fig. 7 is considered. Geometric parameters and material properties are given as: $E=3.10275 \mathrm{kN} / \mathrm{mm}^{2}, v=0.3, R=2540 \mathrm{~mm}$, straight-line length $L=254 \mathrm{~mm}, \theta=0.1 \mathrm{rad}, h=12.7 \mathrm{~mm}$. At the symmetric center of the cylindrical shell, a concentrated vertical load acts at the point. Because of symmetry, again only one quarter of the structure is taken into consideration. This considered structure is modeled consecutively by $8 \times 8$ (128 elements) meshe. Fig. 8 shows the central deflection of the circular cylindrical shell. As can be found, those nonlinear behaviors from using the three different geometric stiffness matrices are the same and the analysis results are in accordance with those of Ref. [6].

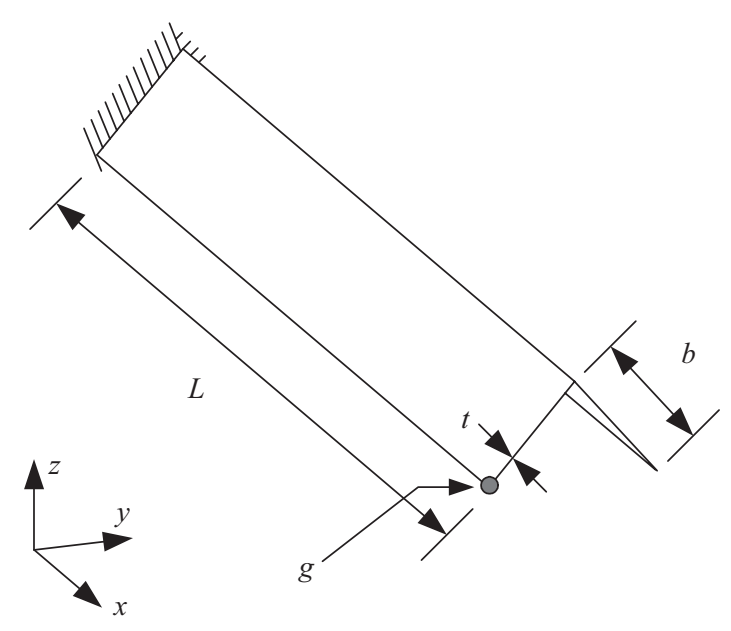

(a) An Illustration of the cantilever beam with $L$ section.
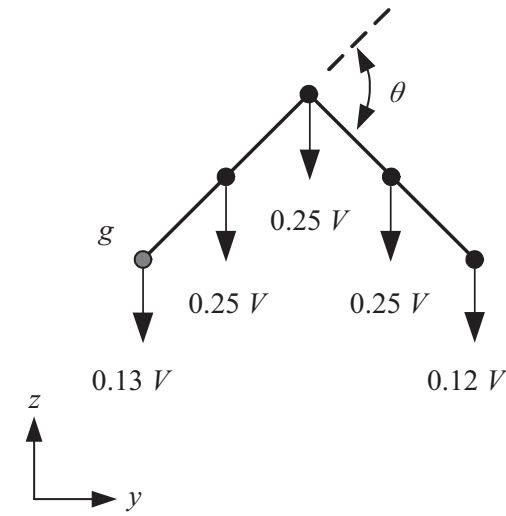

$0.12 V$

(b) An illustration of the nodal forces at the free end.

Fig. 9. A cantilever beam of an $L$ section subjected to the shear force at the end.

\section{Example 2:}

A cantilever beam of a L-type section subjected to a shear force $\mathrm{V}$ at the free end, is shown in Fig. 9, and its material properties and geometrics are as follows: $E=3.10275 \mathrm{kN} / \mathrm{mm}^{2}$, $v=0.3$, beam length $L=1,000 \mathrm{~mm}$, flange thickness $b=100$ $\mathrm{mm}$, plate thickness $h=5 \mathrm{~mm}$, angle of flanges $\theta=\pi / 2$. This considered structure is modeled consecutively by $20 \times 4(160$ elements) meshes. When the structure is subjected to the shear force, it will result in the torsional buckling deformation. Now, consider the unstable behavior at the g point from y direction at the end of the cantilever beam, as shown in Fig. 10, the solid line, which represents the relation of the applied force and the displacement, is the result obtained by using the proposed geometrically nonlinear theory of passing the rigid body rule; while the curve with the symbol " + " represents the result by using the conventional geometrically nonlinear theory; the curve with the symbol "O" represents the result from the use of the simplified theory, which only considers the plate subjected to the membrane force. As shown in this figure, the 




Fig. 10. The displacement of the $y$-axis at the node $a$ in the end of the cantilever beam.

critical loadings of the latter two cases are $10 \%$ differences to that of the former case.

\section{CONCLUSIONS}

In this paper, a complete stability theory for the Krichhoff thin plate has been developed by using the virtual work method. This theory carefully considers various actions, such as three membrane forces per unit length $\left(N_{x x}, N_{y y}\right.$, and $\left.N_{x y}\right)$, moments per unit length $\left(M_{x x}, M_{y y}\right.$, and $\left.M_{x y}\right)$, and transverse shear forces per unit length $\left(Q_{x}\right.$ and $\left.Q_{y}\right)$. Expressing terms for the derived incremental virtual work equation in this plate buckling theory are all written in the explicit forms and every term in this theory has its own physical meaning. In addition, a rigid body test especially for the incremental virtual work equation has been constructed and the proposed theory can pass the rigid body test successfully. In practice, the geometric stiffness matrix derived from the current approach can consider various actions and pass the rigid body test automatically; therefore, the derived stiffness matrix, based on the current theory, can correctly analyze the buckling and nonlinear behaviors for the plate and shell structure.

\section{ACKNOWLEDGMENTS}

The first author would like to express his thank to the National Science Council, Taiwan for their financial support under Grant Number: NSC 93-2211-E-019-009. In addition, his thank also goes to Prof. Weichung Yeih and Prof. JinagRen Chang for some valuable discussions on this work.

\section{APPENDIX I}

Using the boundary forces in the ${ }^{2} C$ state in (32), the first integration term in the right hand side of (44) can be written as

$$
\begin{aligned}
& \oint_{2_{s}}\left(\int_{-t / 2}^{t / 2}\left\{\begin{array}{c}
{ }^{2} t_{x} \\
{ }^{2} t_{y} \\
{ }^{2} t_{z}
\end{array}\right\}^{T} d \xi\right)\left\{\begin{array}{l}
\delta u \\
\delta v \\
\delta w
\end{array}\right\} d^{2} s \\
& =\oint_{{ }^{2} s}\left[{ }^{2} N_{x} \delta u+{ }^{2} N_{y} \delta v+{ }^{2} N_{z} \delta w\right] d^{2} s \\
& -\oint_{2_{s}}\left[\frac{\partial}{\partial^{2} s}\left({ }^{2} M_{\beta} \theta_{y}\right) \delta u-\frac{\partial}{\partial^{2} s}\left({ }^{2} M_{\beta} \theta_{x}\right) \delta v\right. \\
& \left.+\frac{\partial}{\partial^{2} s}\left({ }^{2} M_{\beta}\right) \delta w\right] d^{2} s \text {. }
\end{aligned}
$$

The second integration term appearing in (A1) using integration by parts, we have

$$
\begin{aligned}
\oint_{2_{s}}\left[\frac{\partial}{\partial^{2} s}\right. & \left({ }^{2} M_{\beta} \theta_{y}\right) \delta u-\frac{\partial}{\partial^{2} s}\left({ }^{2} M_{\beta} \theta_{x}\right) \delta v \\
& \left.+\frac{\partial}{\partial^{2} s}\left({ }^{2} M_{\beta}\right) \delta w\right] d^{2} s \\
= & -\oint_{2_{s}}{ }^{2} M_{\beta}\left[\theta_{y} \frac{\partial(\delta u)}{\partial^{2} s}-\theta_{x} \frac{\partial(\delta v)}{\partial^{2} s}+\frac{\partial(\delta w)}{\partial^{2} s}\right] d^{2} s
\end{aligned}
$$

Using the definition for rotational angles in (10) and (11) and the relationship for edge lengths in (19) and (22), we have

$$
\begin{aligned}
\theta_{y} & \frac{\partial}{\partial^{2} s} \delta u-\theta_{x} \frac{\partial}{\partial^{2} s} \delta v+\frac{\partial}{\partial^{2} s} \delta w \\
= & \frac{d^{1} s}{d^{2} s}\left(\theta_{y} \frac{\partial}{\partial^{1} s} \delta u-\theta_{x} \frac{\partial}{\partial^{1} s} \delta v+\frac{\partial}{\partial^{1} s} \delta w\right) \\
& =\frac{1}{\left(1+e_{s s}\right)}\left(-\theta_{s} \delta \theta_{z}^{*}-\theta_{n} \delta e_{s s}+\delta \theta_{n}\right) \\
& \approx \delta \theta_{n}-e_{s s} \delta \theta_{n}-\theta_{n} \delta e_{s s}-\theta_{s} \delta \theta_{z}^{*} .
\end{aligned}
$$

Introducing (A2) and (A3), Eq. (A1) can be rewritten as

$$
\begin{aligned}
& \oint_{2_{s}}\left(\int_{-1 / 2}^{1 / 2}\left\{\begin{array}{c}
{ }^{2} t_{x} \\
{ }^{2} t_{y} \\
{ }^{2} t_{z}
\end{array}\right\}^{T} d \xi\right)\left\{\begin{array}{l}
\delta u \\
\delta v \\
\delta w
\end{array}\right\} d^{2} s \\
& =\oint_{2_{s}}\left[{ }^{2} N_{x} \delta u+{ }^{2} N_{y} \delta v+{ }^{2} N_{z} \delta w+{ }^{2} M_{\beta} \delta \theta_{n}\right] d^{2} s \\
& \quad-\oint_{2_{s}}{ }^{2} M_{\beta}\left(e_{s s} \delta \theta_{n}+\theta_{n} \delta e_{s s}+\theta_{s} \delta \theta_{z}^{*}\right) d^{2} s
\end{aligned}
$$

Now decompose the traction in the ${ }^{2} C$ state,${ }^{2} t_{i}$, as the sum of the traction in the ${ }^{1} C$ state, ${ }^{1} t_{i}$, and the incremental traction $\left(\Delta t_{i}\right)$, that is 


$$
\left\{\begin{array}{l}
{ }^{2} t_{n} \\
{ }^{2} t_{s} \\
{ }^{2} t_{z}
\end{array}\right\}=\left\{\begin{array}{l}
{ }^{1} t_{n} \\
{ }^{t_{s}} \\
{ }^{{ }} t_{z}
\end{array}\right\}+\left\{\begin{array}{l}
\Delta t_{n} \\
\Delta t_{s} \\
\Delta t_{z}
\end{array}\right\}
$$

Adopting the transformation of coordinate systems in (18), ${ }^{2} t_{\alpha},{ }^{2} t_{\beta}$ and ${ }^{2} t_{\gamma}$ can be expressed as

$$
\begin{aligned}
{ }^{2} t_{\alpha} & ={ }^{2} t_{n}+{ }^{2} t_{s} \theta_{z}^{*}-{ }^{2} t_{z} \theta_{s} \\
& ={ }^{1} t_{n}+\left[\Delta t_{n}+\left({ }^{1} t_{s}+\Delta t_{s}\right) \theta_{z}^{*}-\left({ }^{1} t_{z}+\Delta t_{z}\right) \theta_{s}\right], \\
{ }^{2} t_{\beta} & =-{ }^{2} t_{n} \theta_{z}^{*}+{ }^{2} t_{s}+{ }^{2} t_{z} \theta_{n} \\
& ={ }^{1} t_{s}+\left[-\left({ }^{1} t_{n}+\Delta t_{n}\right) \theta_{z}^{*}+\Delta t_{s}+\left({ }^{1} t_{z}+\Delta t_{z}\right) \theta_{n}\right], \\
{ }^{2} t_{\gamma} & ={ }^{2} t_{n} \theta_{s}-{ }^{2} t_{s} \theta_{n}+{ }^{2} t_{z} \\
& ={ }^{1} t_{z}+\left[\left({ }^{1} t_{n}+\Delta t_{n}\right) \theta_{s}-\left({ }^{1} t_{s}+\Delta t_{s}\right) \theta_{n}+\Delta t_{z}\right],
\end{aligned}
$$

respectively. Multiplying (A6) to (A8) with $\xi$, and integrating with respect to the plate thickness and then, introducing the definitions for the boundary moments per unit length in (13) and (28), we obtain

$$
\begin{aligned}
{ }^{2} M_{\alpha}= & \int_{-h / 2}^{1 / 2} t_{n} \xi d \xi \\
& +\int_{-1 / 2 / 2}^{1 / 2}\left[\Delta t_{n}+\left({ }^{1} t_{s}+\Delta t_{s}\right) \theta_{z}^{*}-\left({ }^{1} t_{z}+\Delta t_{z}\right) \theta_{s}\right] \xi d \xi \\
= & M_{n}+\underline{\int_{-n / 2}^{1 / 2}\left[\Delta t_{n}+\left({ }^{1} t_{s}+\Delta t_{s}\right) \theta_{z}^{*}-\left({ }^{1} t_{z}+\Delta t_{z}\right) \theta_{s}\right] \xi d \xi,}
\end{aligned}
$$

$$
\begin{aligned}
{ }^{2} M_{\beta}= & \int_{-n / 2}^{n / 2} t_{s} \xi d \xi \\
& +\int_{-n / 2}^{1 / 2}\left[-\left({ }^{1} t_{n}+\Delta t_{n}\right) \theta_{z}^{*}+\Delta t_{s}+\left({ }^{1} t_{z}+\Delta t_{z}\right) \theta_{n}\right] \xi d \xi \\
= & { }^{1} M_{s}+\underline{\int_{-n / 2}^{1 / 2}\left[-\left({ }^{1} t_{n}+\Delta t_{n}\right) \theta_{z}^{*}+\Delta t_{s}+\left({ }^{1} t_{z}+\Delta t_{z}\right) \theta_{n}\right] \xi d \xi,}
\end{aligned}
$$

$$
\begin{aligned}
\int_{-n / 2}^{n / 2} t_{\gamma} \xi d \xi & =\int_{-n / 2}^{n / 2} t_{z} \xi d \xi \\
& +\int_{-n / 2 L}^{n / 2}\left[\left({ }^{1} t_{n}+\Delta t_{n}\right) \theta_{s}-\left({ }^{1} t_{s}+\Delta t_{s}\right) \theta_{n}+\Delta t_{z}\right] \xi d \xi .
\end{aligned}
$$

Since the incremental tractions and incremental displacements are tiny quantities, the underlined terms in (A9) to (A11) is relatively smaller in an order than the first terms appearing in the right hand sides of equations. Substituting (A10) into the second integration terms in (A4) and neglecting the higher order terms can yield

$$
\begin{aligned}
& \oint_{2_{s}}^{2} M_{\beta}\left(e_{s s} \delta \theta_{n}+\theta_{n} \delta e_{s s}+\theta_{s} \delta \theta_{z}^{*}\right) d^{2} s \\
& \approx \oint_{1_{s}}^{1} M_{s}\left(e_{s s} \delta \theta_{n}+\theta_{n} \delta e_{s s}+\theta_{s} \delta \theta_{z}^{*}\right) d^{1} s .
\end{aligned}
$$

Substituting (A12) into (A4), Eq. (45) then can be obtained.

\section{APPENDIX II}

Substituting (26) and (27) into the second integration terms in the right hand side of (44) yields

$$
\begin{aligned}
\oint_{2_{s}}\left(\int_{-h / 2}^{h / 2}\left\{\begin{array}{c}
{ }^{2} t_{n} \\
2^{2} t_{s}
\end{array}\right]^{T} \xi d \xi\right)\left\{\begin{array}{c}
\delta \theta_{s} \\
-\delta \theta_{n}
\end{array}\right\} d^{2} s . \\
=\oint_{2_{s}}\left({ }^{2} M_{\alpha} \delta \theta_{s}-{ }^{2} M_{\beta} \delta \theta_{n}\right) d^{2} s \\
\quad-\oint_{2_{s}}\left({ }^{2} M_{\alpha} \theta_{z} \delta \theta_{n}+{ }^{2} M_{\beta} \theta_{z}^{*} \delta \theta_{s}\right) d^{2} s \\
+\oint_{2_{s}}\left(\int_{-h / 2}^{h / 2} t_{\gamma} \xi d \xi\right)\left(\theta_{s} \delta \theta_{s}+\theta_{n} \delta \theta_{n}\right) d^{2} \mathrm{~s} .
\end{aligned}
$$

Substituting (A9) and (A10) into the second integration terms in the right hand side of (A13) and neglecting the higher order terms, we have

$$
\begin{aligned}
& \oint_{2_{s}}\left({ }^{2} M_{\alpha} \theta_{z} \delta \theta_{n}+{ }^{2} M_{\beta} \theta_{z}^{*} \delta \theta_{s}\right) d^{2} s \\
& \quad \approx \oint_{1_{s}}\left({ }^{1} M_{n} \theta_{z} \delta \theta_{n}+{ }^{1} M_{s} \theta_{z}^{*} \delta \theta_{s}\right) d^{1} s .
\end{aligned}
$$

Substituting (A11) into the third integration term in (A13) and neglecting the higher order terms, we have

$$
\begin{aligned}
& \oint_{2_{s}}\left(\int_{-n / 2}^{n / 2} t_{\gamma} \xi d \xi\right)\left(\theta_{s} \delta \theta_{s}+\theta_{n} \delta \theta_{n}\right) d^{2} \mathrm{~s} \\
& \quad \approx \oint_{1_{s}}\left(\int_{-n / 2}^{n / 2} t_{z} \xi d \xi\right)\left(\theta_{s} \delta \theta_{s}+\theta_{n} \delta \theta_{n}\right) d^{1} \mathrm{~s} .
\end{aligned}
$$

Substituting (A14) and (A15) into (A13), Eq. (46) can be yielded.

\section{REFERENCES}

1. Bathe, K. J., Finite Element Procedutes in Engineering Analysis, Prentice-Hall, (1982). 
2. Batoz, J. L., Bathe, K. J., and Ho, L. W., "A study of three-node triangular plate bending element," International Journal for Numerical Methods in Engineering, Vol. 15, pp. 1771-1812 (1980).

3. Chajes, A., Principles of Structural Stability Theory, Prentice-Hall, Upper Saddle River, NJ (1974).

4. Cook, R. D., Malkus, D. S., and Plesha, M. E., Concepts and Applications of Finite Element Analysis, 3rd Ed., John Wiley \& Sons, Hoboken, NJ (1989).

5. Hodges, D. H., Atilgan, A. R., and Danielson, D. A., “A geometrically nonlinear theory of elastic plates," Journal of Applied Mechanics, ASME, Vol. 60, pp. 109-116 (1993).

6. Horrigome, G. and Bergan, P. G., "Nonlinear analysis of free-form shell by flat finite elements," Computer Methods in Applied Mechanics and Engineering, Vol. 16, pp. 11-35 (1987).

7. Kuo, S. R., Chi, C. C., Yeih, W., and Chang, J. R., "A reliable three-node triangular plate element satisfying rigid body rule and incremental force equilibrium condition," Journal of the Chinese Institute of Engineers, Vol. 29, No. 4, pp. 619-632 (2006).

8. Kuo, S. R. and Yang, Y. B., "Tracing postbuckling paths of structures containing multi-loops," International Journal for Numerical Methods in Engineering, Vol. 38, No. 23, pp. 4053-4075 (1995).

9. Platt, J., Davies, G., and Snell, C., "Critical review of thin-plate stability equation," Journal of Engineering Mechanics, ASCE, Vol. 118, No. 3, pp. 481-495 (1992).

10. Timoshenko, S. P. and Gere, J. M., Theory of Elastic Stability, 2nd Ed., McGraw-Hill, New York, NY (1961).

11. Timoshenko, S. P. and Woinowsky-Krieger, S., Theory of Plates and Shells, 2nd Ed., McGraw-Hill, New York, NY (1959).

12. Ugural, A. C., Stresses in Plates and Shells, McGraw-Hill, New York, NY (1981).
13. Wang, C. M., Kitipornchai, S., and Xiang, Y., "Relationships between buckling loads of Kirchhoff, Mindlin, and Reddy polygonal plates on pasternak foundation," Journal of Engineering Mechanics, ASCE, Vol. 123, No.11, pp.1134-1137 (1997).

14. Wempner, G., Mechanics of Solids with Applications to Thin Bodies, Sijthoff \& Noordhoff, Amsterdam, Netherlands (1981).

15. Wsshizu, K., Variational Method in Elasticity and Plasticity, 2nd Ed., Pergamon Press, Oxford, UK (1975).

16. Xiang, Y., "Exact solutions for buckling of multispan rectangular plates," Journal of Engineering Mechanics, ASCE, Vol. 129, No. 2, pp. 181-187 (2003).

17. Yang, Y. B., Chang, J. T., and Yau, J. D., “A simple nonlinear triangular plate element and strategies of computation for nonlinear analysis," Computer Methods in Applied Mechanics and Engineering, Vol. 178, pp. 307-321 (1999).

18. Yang, Y. B. and Chiou, H. T., "Rigid body motion test for nonlinear analysis with beam elements," Journal of Engineering Mechanics, ASCE, Vol. 113, No. 9, pp.1404-1419 (1987).

19. Yang, Y. B. and Kuo, S. R., Theory and Analysis of Nonlinear Framed Structures, Prentice Hall, Upper Saddle River, NJ (1994).

20. Yang, Y. B. and Shieh, M. S., "Solution method for nonlinear problems with multiple critical points," American Institute of Aeronautics and Astronautics Journal, Vol. 28, No. 12, pp. 2100-2116 (1990).

21. Yang, Y. B., Yau, J. D., and Kuo, S. R., "Theory of stability for straight solid beams that passes the rigid body test," Bulletin of College of Engineering, N.T.U., No. 70, pp. 1-23 (1997).

22. Zhang, J. W. and Shen, H. S., "Postbuckling of orthotropic rectangular plates in biaxial compression." Journal of Engineering Mechanics, ASCE, Vol. 117, No. 5, pp. 1158-1170 (1991).

23. Ziegler, H., Principles of Structural Stability, 2nd Ed., Birkhäuser, Basel, Switzerland (1977). 